\title{
THE EFFECT OF ATMOSPHERIC PLASMA-SPRAYED PEEK IMPLANTS ON OSSEOINTEGRATION
}

\author{
Aliaa Mahrous*, Samah Mohamed** and Alshaimaa Ahmed ${ }^{* * *}$
}

\begin{abstract}
Objectives: The study evaluated the osseointegration of atmospheric plasma treated PEEK implant surfaces versus the untreated PEEK surfaces.

Material \& Methods: The PEEK implants were milled using carbon reinforced PEEK material with CAD-CAM milling machine. The implants were placed in 6 male mongrel dogs, each dog received 2 groups of PEEK implants. The control group consisted of untreated surface PEEK implants. The study group consisted of atmospheric plasma-sprayed PEEK implants. the implants were placed in the dog femur. After 12 weeks, the animals were sacrificed, and boneimplant sections were obtained for histological evaluations and bone-to-implant contact. Electron microscope scanning was performed to assess the topography of the surface of treated and untreated PEEK implant surface.
\end{abstract}

Results: Qualitative evaluation of the stained histological sections of the PEEK implants demonstrated a well distributed bone in proximity to the atmospheric plasma-sprayed PEEK implant surface after 12 weeks of placements. The bone-to-implant contact showed a significant difference $(\mathrm{P}<0.001)$ between treated and untreated PEEK implant surface.

Conclusions: Within the limitation of the present study, it is concluded that the atmospheric plasma-sprayed surface treatment may improve the osseointegration of PEEK implant.

KEY WORDS: PEEK, Osseointegration, plasma-spray, titanium, dental implant.

\section{INTRODUCTION}

Dental implants had become the standard of care to replace the missing teeth They improve the patient's quality of life by restoring the normal contour, function, comfort, esthetics, speech, and health With the spread use of the dental implants, many research had been directed toward improving the long-term success and improving the quality of the treatment provided ${ }^{(1-4)}$

\footnotetext{
* Lecturer of fixed prosthodontic, Faculty of Dentistry, Fayoum University, Egypt

** Lecturer of Oral Biology, Faculty of Dentistry, MSA University, Egypt

*** Lecturer of Oral \& Maxillofacial Surgery, Faculty of Dentistry, Fayoum University, Egypt
} 
For decades in orthopedic, as well as oral and maxillofacial surgery, metallic implants made of titanium (Ti) and its alloys were the main choice as an implant material as they provide sufficient mechanical strength and excellent friction resistance for permanent prostheses and implants ${ }^{(5,6)}$

Although implants based on titanium and titanium alloys, it showed that their use can be correlated with a range of problems ${ }^{(7,8)}$ One of the main problems is the difference of Young's moduli between bone and titanium The Young's moduli of most Titanium alloys (116 GPa) are generally much higher and do not match those of normal cortical human bone tissues (17.7 GPa) The redistributed and decreased loading on the bone causes a stress shielding effect with reduction of bone density of adjacent bone tissues with the risk of subsequent loosening or failure of the implant ${ }^{(9,10)}$

Moreover, metallic implants had inherent high radiopacity which may generate scattering artifacts in computed tomography (CT) images This poses the challenge to identify any initial peri-implant resorption or loss of bone mineral density ${ }^{(11)}$ Also, titanium can cause esthetic problems due to its color and lack of light transmission This can provoke a dark shimmer of the peri-implant soft tissue especially in cases with a thin biotype mucosa and mucosa recession around a titanium implant This can be a problem especially in the presence of a high smile line ${ }^{(12,13)}$ Furthermore, many studies had documented cases of patients developing hypersensitivity to titanium dental implants ${ }^{(14,15)}$

To overcome the disadvantages and minimize the post-implantation biological reactions, substitutes for metal dental implants had been proposed As an alternative, ceramic dental implants made of zirconia had been introduced Zirconia implants seem to be a better alternative because of its tooth-like color, mechanical properties, biocompatibility, and low plaque affinity However, the stress distribution of a zirconia implant to the surrounding bone could be associated with even higher stress peaks compared to titanium, due to the higher elastic modulus of zirconia of $210 \mathrm{GPa}{ }^{(12,16,17)}$

Another biocompatible alternative, Polyetheretherketone (PEEK) is presented as the prime candidate to replace the metallic implant made of titanium and its alloys PEEK is an organic semicrystalline thermoplastic possessing high thermal stability, good fracture resistance, and excellent chemical resistance Furthermore, it is also radiolucent and can be repeatedly sterilized and shaped by machining and heat contouring ${ }^{(18-20)}$

The major beneficial property for implant application remains its lower Young's (elastic) modulus (3- $4 \mathrm{GPa}$ ) being close to human bone PEEK can be modified easily by incorporation of other materials to increase the strength of the material For example; incorporation of carbon fibers can increase the elastic modulus up to $18 \mathrm{GPa}$ which is also comparable to those of cortical bone so the polymer could exhibit lesser stress shielding when compared to titanium which used as an implant material ${ }^{(19,21)}$

PEEK can be used for many applications in dentistry including dental implants, abutments, and framework for fixed and removable partial dentures ${ }^{(19)}$ In contrast to titanium, PEEK has very limited inherent osteoconductive properties Considerable amount of research had been conducted to improve the bioactivity of PEEK implants There are several methods that have been advocated to improve the bioactivity of PEEK including coating PEEK with synthetic osteoconductive hydroxyl apatite, increasing its surface roughness and chemical modifications and incorporating bioactive particles ${ }^{(22-30)}$

One of the methods that is usually used to increase the bioactivity of the PEEK was plasma spraying This method had many drawbacks as it deposited very thick apatite layer that may get delaminated leading to implant failures Another disadvantage of using 
plasma-spray to coat PEEK with hydroxyapatite are the high temperature involved in the process High temperatures could damage PEEK structure due to its relatively low melting temperature (ca 340 8C) Moreover, the low bond strength of plasma-sprayed hydroxyapatite coatings on CFR-PEEK has been suggested to result from the evaporation of carbon fibers from the surface of the implant due to the high temperatures during the coating process $(28,31,32)$

Another technique of plasma generation occurs at ambient temperatures and atmospheric pressures, termed atmospheric pressure plasma (APP) treatment In this process, promoting the formation of reactive compounds on the implant surface Previous studies have demonstrated enhanced osseointegration through atmospheric pressure plasma treatment on the titanium surfaces ${ }^{(33-36)}$

Although several studies had been advocated for modification of PEEK surface, no studies so far had studied the effect of atmospheric plasma treatment on the osseointegration of the PEEK The aim of the current study was to evaluate the osseointegration of atmospheric plasma treated PEEK implant surfaces versus the untreated PEEK surfaces

\section{MATERIAL AND METHODS}

\section{Milling CFR-PEEK Implant:}

Dental Implant (TRI ${ }^{\circledR}$ implants, TRI Dental implants, Bösch, Germany) of $3.3 \mathrm{~mm}$ diameter and $10 \mathrm{~mm}$ length was scanned using a bench scanner (Yenascan E7, Yenadent, Verizon, France) After the scanning process, the STL file was exported and a CAD model was constructed using software (Yenadent CAM, Yenadent, Verizon, France) This CAD model was the basis for milling the carbon fiber reinforced (CFR) PEEK implants All implants were fabricated with the same equipment and materials by an experienced dental technician A new set of milling burs was used after each implant milling procedure A total of 12 CFR-PEEK implants were milled from a CFR-PEEK blocks (JUVORA ${ }^{\mathrm{TM}}$ Dental Disc, JUVORA Ltd, Wyre, Lancashire, UK) in a 5 -axis +1 milling machine (Yenadent D405axis, Yenadent, Verizon, France).

After milling, the implants were cleaned in buffered water using ultrasound to aid in removal of any residuals After drying, the implants were inserted in a sterile barrier packing The implants were sterilized using Gamma irradiation, receiving a dose between 25-35 kGy.

\section{Animal \& Surgical Procedures:}

The present study was conducted on 6 skeletally mature mongrel male dogs Each dog was given a complete clinical, physical and radiographic examination to exclude any evidence of systemic, orthopedic and neurologic diseases Dogs included in the study were with a $15 \pm 1.7 \mathrm{~kg}$ body weight and average age $20 \pm 2.3$ months The animals were housed and quarantined individually in separate cages at the department of surgery, anesthesiology, and radiology, Faculty of veterinary medicine, Cairo University for one week to become acclimatized to the housing and diet.

All animal experiments and surgical procedures were conducted according to the laws of animal protection and welfare and all the study procedures were approved by the ethical committee of Faculty of Veterinary Medicine- Cairo University.

The animals were fastened for $12 \mathrm{hrs}$ prior to surgery and allow free access to drinking water till time of anesthesia The animals were prepared in lateral recumbency with the right side uppermost the skin over the right pelvic limb from dorsal midline to the tarsal joint were prepared for aseptic surgery The hair coat was clipped and shaved, and disinfected with povidone iodine 10\% (Betadine, Nile pharm, Egypt) (Fig 1)

An intramuscular injection of ketamine 10\%,(10 $\mathrm{mg} / \mathrm{kg})\left(\right.$ Ketamax ${ }^{\circledR}$, Gujarat, India) and Xylazine 2\%, (1 mg/kg) (Xylaject ${ }^{\circledR}$, ADWIA Co S.A.E., 
$10^{\text {th }}$ of Ramadan city, Egypt), was used to sedate the animals prior to operation The IV injection Sodium thiopental 5\% (Thipen $\AA$, Sigma, Egypt) with average dose $(10 \mathrm{mg})$ was used for general anesthesia The respiratory air way was kept patent by applying an endotracheal tube.

The animal was fixed on operating table in lateral position A lateral approach to the right femur was done through a skin incision along the craniolateral border of the shaft of the bone from the level of the greater trochanter to the level of patella The skin margin was undermined and retracted and the superficial leaf of the fascia lata was incised along the entire length of the skin incision Caudal retraction of the biceps fumoirs revealed the shaft of the femur The periosteum around the osteotomy site was reflected using periosteal elevators

Osteotomy was performed with a series of drills under external continuous irrigation until the final diameter of $3.2 \mathrm{~mm}$ was reached Two implants (untreated CFR-PEEK, atmospheric pressure plasma CFR-PEEK) were placed along femur The implants were press fit into $3.2 \mathrm{~mm}$ drill holes by hand pressure until they were fully submerged into bone They were placed from proximal to distal directions, approximately $4 \mathrm{~cm}$ apart

Prior to implant insertion, the experimental set of implants was treated with an atmospheric pressure plasma device (Piezobrush ${ }^{\circledR}$ PZ2, Relyon plasma GMBH, Regensburg, Germany) for a total of 80 seconds (20 seconds per implant quadrant)

Closure was achieved with standard layered suturing techniques with VICRYL 4-0 (Ethicon Johnson, Miami, FL) for deep tissues and nylon 4-0 (Ethicon Johnson, Miami,FL) for skin The dogs remained in the animal care facility and received antibiotics (benzyl penicillin benzathine $20.00 \mathrm{IU} /$ $\mathrm{kg}$ ) and anti-inflammatory medication (ketoprofen $1 \%, 1 \mathrm{~mL} / 5 \mathrm{~kg}$ ) for pain control (Fig.1)

The site of surgery of the animals was examined radiographically immediately after operation and at interval of 4,8 and 12 weeks throughout the study After 12 weeks of healing, the animals were sacrificed with an overdose of sodium pentobarbital $(60 \mathrm{mg} / \mathrm{ml})$ The femur was retrieved, and soft tissue were removed Implants and adjacent bone (at least $1 \mathrm{~cm}$ ) were separated into small blocks.

\section{Histological Preparation:}

After harvesting the specimen, the specimens had been kept in $4 \%$ formaldehyde for 1 day The specimens were then dehydrated in an ascending series of ethanol concentrations (60-100\%) and then embedded in transparent chemically polymerized methyl methacrylate (99\%; Sigma-Aldrich, Steinheim, Germany) Following polymerization, nondecalcified thin longitudinal sections of the specimens (three middle sections of each specimen, parallel to the long axis of the implants) were cut using a microsectioning system with a rotary diamond coated saw cooled with running water (Micracut 150 precision cutter, Metkon, Bursa,Turkey) Subsequently, the ground sections were prepared and polished with 800-grit silicon carbide paper, and then stained with toluidine blue

The sections were observed under a light microscope (Olympus BX61, Hamburg, Germany) connected to a high-resolution digital camera (Olympus, E330, Imaging Corp), whereby digital images were obtained for each section and the BIC\% was measured as the percentage of bone in direct contact with the implant surface.

On each specimen, one of the section was Gold sputtering in a sputter coater (K550 Sputter Coater, EMITEC, England) The sputtered sampled were examined under SEM (Model Quanta 250 FEG, FEI company, Netherlands) attached with EDX Unit (Energy Dispersive X-ray Analyses), with accelerating voltage $30 \mathrm{~K} . \mathrm{V}$., to evaluate the gap distance between the implant surface and bone The results were recorded photographically using magnifications up to 500x. 


\section{Scanning electron microscope analysis:}

The surface topography of CFR-PEEK implant before and after (APP) treatment was examined using SEM with magnifications up to $30000 \mathrm{x}$ Energy Dispersive X-ray Analyses used to evaluate the changes in the chemical changes on the implant surface before and after (APP) treatment The surface roughness of the samples was record using Scandium software (Scandium, Scandium Olympics soft image solution $\mathrm{GmbH}$, USA)

\section{Statistical analysis}

Data were fed to the computer and analyzed using IBM SPSS software package version 20.0 Data were fed to the computer and analyzed using IBM SPSS software package version 20.0 (Armonk, NY: IBM Corp) The Kolmogorov- Smirnov, Shapiro and D'agstino tests were used to verify the normality of distribution of variables Mann Whitney test was used to compare between two groups for abnormally distributed quantitative variables Significance of the obtained results was judged at the $5 \%$ level.

\section{RESULT}

\section{Surgery and postoperative period:}

Surgery and anesthesia were uneventful No signs of discomfort or unusual reactions were seen in animals during the operation During the healing period, no deep or superficial infection were recorded All animals showed normal food and water intake 10 days after surgery.

All implants were placed according to the intended protocols and distribution Adapted diameters of drills holes allowed for primary implant stability of all implants and thus no implant exhibited clinical mobility.

\section{Macroscopic and Radiographic evaluation:}

Upon retrieval of the bone and surrounding tissues all sites revealed undisturbed with stable fixation of implants No implants were lost There were no signs of inflammatory or foreign body reaction was visible Visual inspections of the surgical sites revealed a layer of new periosteal bone at the cortical area around two treated implants.

Ventrolateral radiographs showed that all implants were placed correctly and there were no signs of inflammation or any signs of bone resorption around the implant during the follow-up period.

\section{Histological evaluation:}

The histological evaluation revealed that the bone surrounding the implants was a mixture of mature lamellar bone and woven bone close to the surface Areas of a new bone formation, with mainly osteoblasts and remodeling areas exhibiting resorption, were seen (Fig $1 \& 2$ ).

Moreover, close bone-to-implant contact was observed for the treated CFR-PEEK implants while in the untreated CFR-PEEK implants no contact was observed in many areas along the implant surface and a cell-rich tissue impeded the bone formation at the contact with the implant surface Two implant samples of untreated CFR-PEEK implants were separated during sectioning procedures indicating that there was no enough contact between the tooth and implant surface

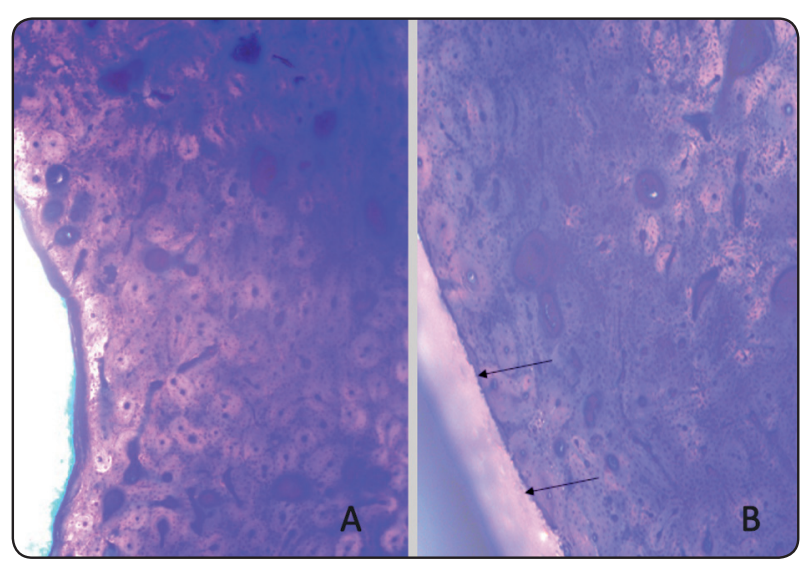

Fig. (1) Photomicrograph showing contact between the implant and bone (black arrows) (toluidine blue X 200) a: untreated CFR-PEEK implant, b: treated CFR-PEEK implant 
The quantitative evaluation of the bone-implant contact revealed less contact between bone and untreated CFR-PEEK implants surface (mean $55.68 \pm 6.4 \%$ ) compared with treated CFR-PEEK implants (mean $88.6 \pm 4.6 \%$ ) The difference between the treated and untreated bone-implant contact showed a statistically significant relation $(\mathrm{p}<0.001)$ (Table 1)

The SEM scanning of the histological sections showed a wider gap distance between the bone and untreated CFR-PEEK implant surface (mean= $40.73 \pm 6.45 \mu \mathrm{m}$ ) while the gap distance between the treated CFR-PEEK implant surface and bone surface (mean $=14.63 \pm 5.25 \mu \mathrm{m}$ ) the gap distances between the treated and untreated CFR-PEEK showed a statistical significant difference $(\mathrm{p}<0.001)$ (Table 1) (Fig 2)

\section{Scanning electron microscope analysis:}

Scanning electron microscopic images of the surface revealed different topographies of the treated and untreated CFR-PEEK implant surfaces In the untreated CFR-PEEK implant, the surface exhibit a relatively smooth surface with a machined surface finish with no distinct features except for parallel grove along the entire surface While in treated CFRPEEK implant, the surface topography showed a crater with superimposed micron-scale features On
TABLE (1) Comparison between treated and untreated CFR-PEEK surface according to different parameters

\begin{tabular}{cccc}
\hline & $\begin{array}{c}\text { Treated } \\
\text { CFR-PEEK } \\
\text { surface }\end{array}$ & $\begin{array}{c}\text { Untreated } \\
\text { CFR-PEEK } \\
\text { Surface }\end{array}$ & p \\
\hline $\begin{array}{c}\text { Bone-implant } \\
\text { contact }\end{array}$ & $88.6 \pm 4.6 \%$ & $55.68 \pm 6.4 \%$ & $<0.001^{*}$ \\
Gap distance & $14.63 \pm 5.25 \mu \mathrm{m}$ & $40.73 \pm 6.45 \mu \mathrm{m}$ & $<0.001^{*}$ \\
\hline
\end{tabular}

Qualitative data were described using number and percent, while normally quantitative data was expressed in mean $\pm S D$, abnormally distributed data was expressed in median (Min. - Max.) *: Statistically significant at $p \leq 0.05$

higher magnification, micron-scale features were more easily distinguished as small terraces and irregular textures (Fig.3 \& 4)

Energy-dispersive $\mathrm{x}$-ray spectroscopy showed a clear distinct in chemical composition between treated and untreated CFR-PEEK samples Both samples showed composition of carbon and oxygen The spectra of the untreated CFR-PEEK surface should insignificant amount of $\mathrm{Ca}, \mathrm{Si}$ and $\mathrm{Al}$ while in the treated CFR-PEEK samples, there was a statistically significant $(\mathrm{p}<\mathbf{0 . 0 0 1})$ increase in both $\mathrm{Ca}, \mathrm{Al}$ and $\mathrm{Si}$ (Fig 5 - Table 2)
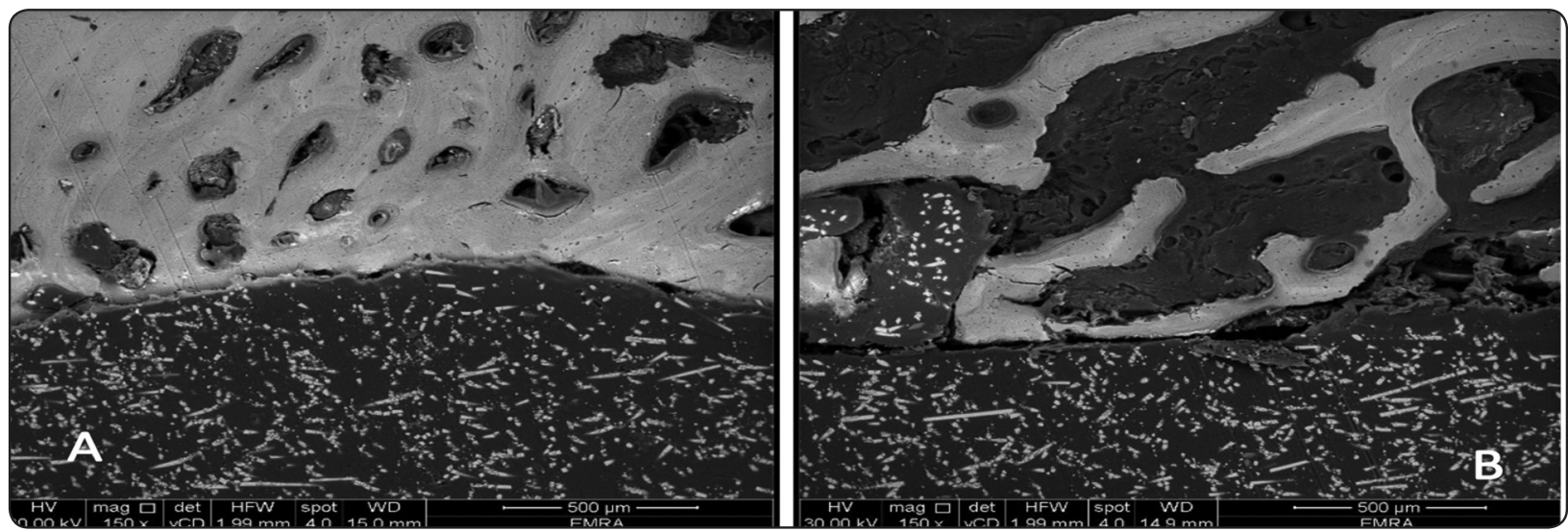

Fig. (2) SEM of histological section (30000x) a: treated CFR-PEEK implant, b: untreated CFR-PEEK implant revealing the difference between the gap distance in the two groups. 


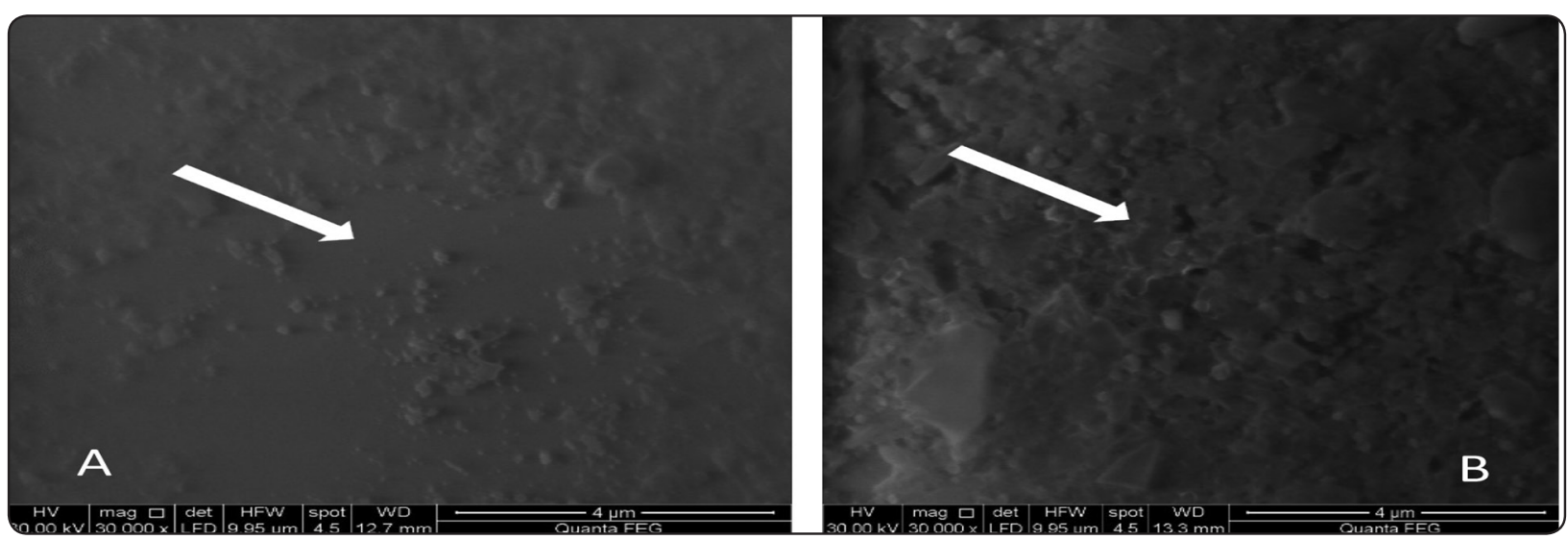

Fig. (3) SEM of the surface topography (30000x) a: untreated CFR-PEEK implant surface, b: treated CFR-PEEK implant surface

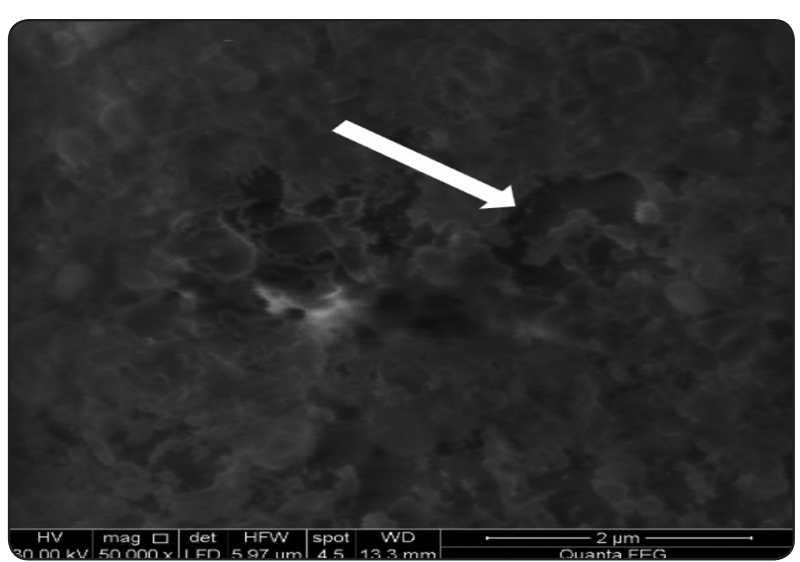

Fig. 4: SEM of the surface topography of treated CFR-PEEK implant surface $(50000 \mathrm{x})$
TABLE (2) Elemental composition from Energy dispersive $\mathrm{X}$-ray of treated and untreated CFR-PEEK implant surface.

\begin{tabular}{lccc}
\hline Atom \% & $\begin{array}{c}\text { Untreated } \\
\text { Surface }\end{array}$ & $\begin{array}{c}\text { Treated } \\
\text { Surface }\end{array}$ & p Value \\
\hline Carbon & $55.35 \pm 2.4$ & $60.45 \pm 3.9$ & $\mathbf{0 . 7 0 8}$ \\
Oxygen & $11.32 \pm 0.34$ & $10.96 \pm 1.69$ & $\mathbf{0 . 8 0 8}$ \\
Ca & $0.65 \pm 0.53$ & $8.8 \pm 0.65$ & $<\mathbf{0 . 0 0 1 *}$ \\
Si & $29.3 \pm 5.3$ & $60.4 \pm 4.66$ & $* \mathbf{0 . 0 0 1 >}$ \\
Al & $8.6 \pm 1.4$ & $12.4 \pm 3.1$ & $<\mathbf{0 . 0 0 1 *}$ \\
\hline
\end{tabular}

Qualitative data were described using number and percent, while normally quantitative data was expressed in mean $\pm S D$, abnormally distributed data was expressed in median (Min - Max.)

*: Statistically significant at $p \leq 0.05$

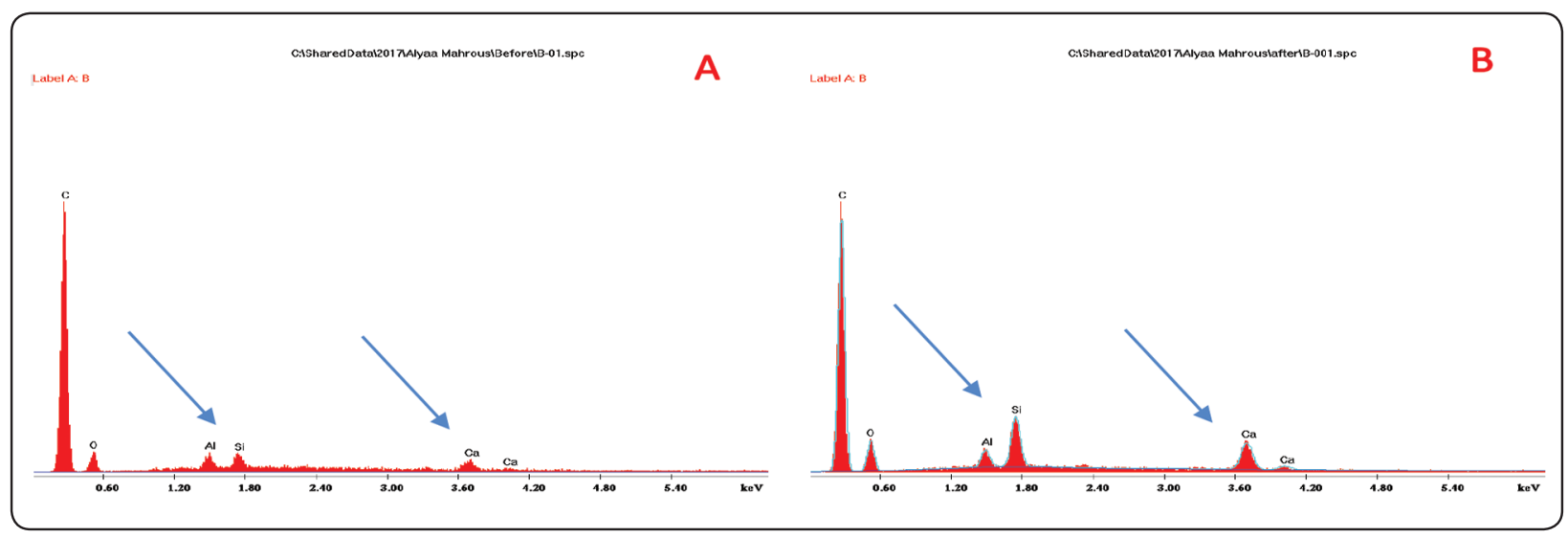

Fig. (5) Energy-dispersive x-ray spectroscopy of a: the untreated CFR-PEEK implant surface, b: the treated CFR-PEEK implant surface. 


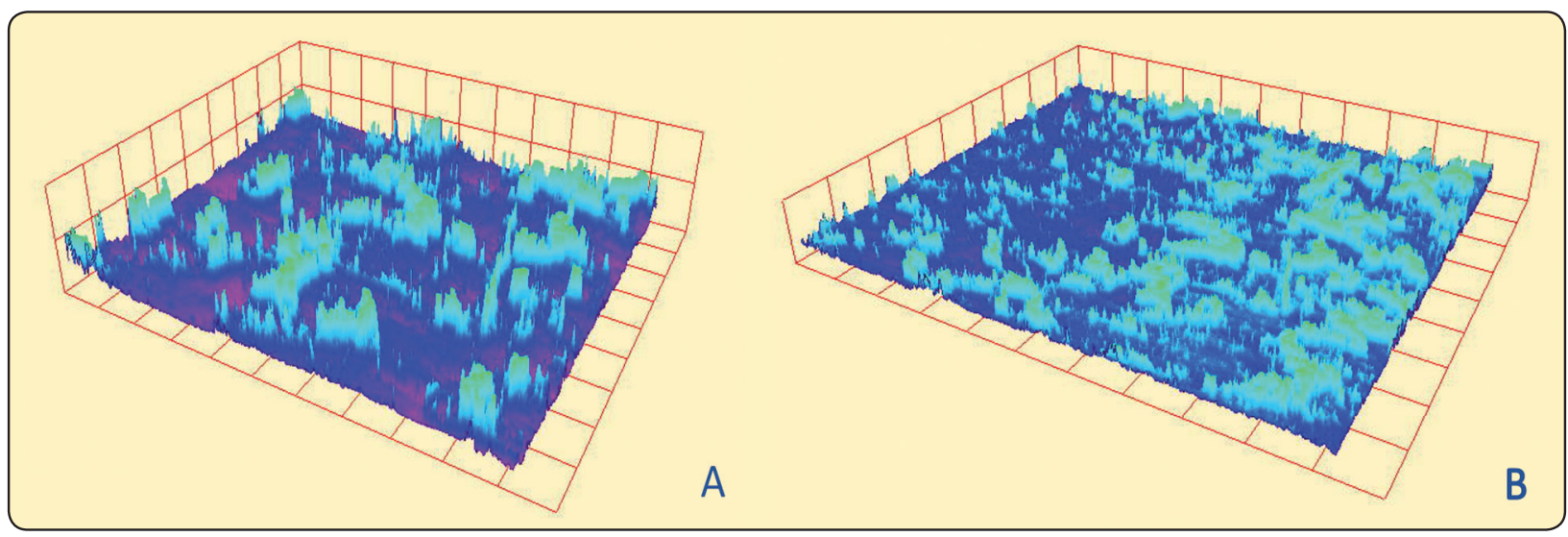

Fig. (6) Surface roughness Diagram of a: the treated CFR-PEEK implant surface, b: the untreated CFR-PEEK implant surface.

Moreover, the plasma treatment also alters the surface roughness between treated and untreated CFR-PEEK implant surface There was increase in the surface roughness value of $360 \pm 3.5$ and 182 \pm 6 in treated and untreated CFR-PEEK implant surface respectively The surface roughness value demonstrated a statistical significant in both treated and untreated CFR-PEEK implant surface (Fig 6)

\section{DISCUSSION}

Osseointegration, the direct structural contact between bone to an implant, is a topic of importance in dental implant success The Surface modifications are directed toward the increase of osseointegration of dental implants and optimizing bone-implant contact This subject was the aim of extensive investigation literature for years ${ }^{(7,18,31-33)}$

Osseointegration is postulated to proceed by adsorption of proteins, which then attract the osteoprogenitor cells onto the implant surface Cellular adhesion could be improved by modification of the implant's surface properties to improve microenvironment on the implant surface for protein adhesion ${ }^{(37-39)}$

Polyetheretherketone (PEEK) is presented as an alternative material to titanium implants PEEK is an organic semicrystalline thermoplastic possessing high thermal stability, good fracture resistance, and excellent chemical resistance Furthermore, it is also radiolucent and can be repeatedly sterilized and shaped by machining and heat contouring ${ }^{(18-20)}$

Unlike the titanium alloys, PEEK has very limited osteoconductive properties Considerable amount of research had been conducted to improve the bioactivity of PEEK implants ${ }^{(22-30)}$ One of the methods that is used to increase the bioactivity of the PEEK was plasma spraying Plasmas operating at atmospheric pressure plasma had been widely used as a tool for the pretreatment of polymers surface ${ }^{(40)}$

The aim of the present study was the utilization of plasma treatment on the implant's surface to improve the osseointegration of CFR- PEEK The study was conducted on the six male mongrel dogs Each dog received 2 groups of CFR-PEEK implants, the untreated CFR-PEEK implant surface served as control group and the atmospheric - pressure plasma treated CFR-PEEK implant served as a study group

Previously in atmospheric pressure plasma generation process, argon gas had been used as an energy carrier that promote the formation of reactive compounds on the implant surface In the present study, the atmospheric pressure plasma used the compressed air as an alternative to argon This was presented as a safe, portable, and cost-effective technology Moreover, the air-based APP treatment improved surface energy of implant by the removal 
of debris or molecules that become accumulated during processing ${ }^{(36)}$

In the present study, the histological evaluation revealed that bone-to-implant contact was observed for the treated CFR-PEEK implants while in the untreated CFR-PEEK implants no contact was observed in many areas along the implant surface and a cell-rich tissue impeded the bone formation at the contact with the implant surface

This in agreement with results of Danna et al ${ }^{(41)}$ who assessed the osseointegrative effects of atmospheric pressure plasma (APP) surface treatment for implants in a canine model They concluded that there was a significant increase in bone to implant for atmospheric pressure plasmatreated textured $\mathrm{Ti}$ surfaces than that of untreated surfaces at 6 weeks postoperative.

Another study by Giro et al ${ }^{(42)}$ investigated the effect of an Argon-based nonthermal plasma surface treatment-operated chairside at atmospheric pressure conditions applied immediately prior to dental implant placement in a canine model They concluded that the argon-based nonthermal plasma improved bone formation around implants at 3 weeks compared with $\mathrm{CaP}$ treatment alone.

The improved osseointegration after the atmospheric pressure plasma treated surface could be largely attributed to the ability of plasma treatment to improve the adhesive bonding for all surfaces either PEEK or titanium surface Moreover, the atmospheric pressure plasma treatment altered the chemical and the topological state of implant surface The observed improvements osseointegration could a result from the combination of these effects $\mathrm{A}$ study by Noeske et al ${ }^{(43)}$ concluded that plasma jet system at atmospheric pressure to improve adhesive bondability to the polymer surfaces and improve the energy level of the surface

In the present study, scanning electron microscope was performed to the treated and untreated surface of CFR-PEEK implant as well as surface roughness and Energy-dispersive x-ray spectroscopy.
In the present study, the results of surface revealed different topographies of the treated and untreated CFR-PEEK implant surfaces In the untreated CFRPEEK implant, the surface exhibit a relatively smooth surface with a machined surface While in treated CFR-PEEK implant, the surface topography showed a crater with superimposed micron-scale features On higher magnification, micron-scale features were more easily distinguished as small terraces and irregular textures

These results are in accordance to the results of Noeske et al ${ }^{(43)}$ who studied the effect of plasma jet on the polymer surface They concluded that the plasma jet increased the surface roughness of the polymer surfaces

The surface roughness of the implant was recognized as the most important determent of the osseointegration of the dental implants Increased surface roughness has enhanced the osteoblast differentiation and local factor production in vitro, as well as bone-toimplant contact and torque removal forces in vivo ${ }^{(44)}$

In the present study, Energy-dispersive x-ray spectroscopy showed statistically significant increase in both $\mathrm{Ca}, \mathrm{Al}$ and $\mathrm{Si}$ in the atmospheric pressure plasma treated CFR-PEEK implant surface The highest increase was in the silica particles

This in agreement with results Noeske et $\mathrm{al}^{(43)}$ that showed that the surface of polymers after plasma treatment increased the percent of $\mathrm{Si}, \mathrm{Ca}$ and oxygen Moreover, Danna et al ${ }^{(41)}$ confirmed the same results in their study of atmospheric pressure plasma treatment.

The increased percent of the silica and calcium on the plasma treated CFR-PEEK implant surface could contribute to the increased bone to implant contact and bone formation at the plasma treated surfaces as silica particles highly improves the osteoblast adhesive responses and stimulates the osteogenic differentiation of stem cells, probably through a mechanic transduction mechanism Several studies have reported a higher rate and extent of bone formation adjacent to implants coated with micro sized bio ceramics ${ }^{(45-47)}$ 


\section{CONCLUSIONS}

Within the limitation of the present study, it is concluded that the atmospheric plasma-sprayed surface treatment may improve the osseointegration of PEEK implants.

\section{Conflict of Interests}

The authors declare that there is no conflict of interests regarding the publication of this paper.

\section{REFERENCES}

1. Adell R, Eriksson B, Lekholm U, Brånemark PI, Jemt T Long-term follow-up study of osseointegrated implants in the treatment of totally edentulous jaws Int J Oral Maxillofac Implants 1990; 5:347-59

2. Bass SL, Triplett RG The effects of preoperative resorption and jaw anatomy on implant success A report of 303cases Clin Oral Implants Res 1991; 2:193-8

3. Becker W, Becker BE, Alsuwyed A, Al-Mubarak S Longterm evaluation of 282 implants in maxillary and mandibular molar positions: a prospective study J Periodontol 1999; 70:896-901

4. Turkyilmaz I, Company AM, McGlumphy EA Should edentulous patients be constrained to removable complete dentures? The use of dental implants to improve the quality of life for edentulous patients Gerodontology 2010; 27:3-10.

5. Saini M, Singh Y, Arora P, Arora V, Jain K Implant biomaterials: A comprehensive review World J Clin Cases 2015; 3:52-7

6. Wang W, Ouyang Y, Poh CK Orthopaedic implant technology: biomaterials from past to future.Ann Acad Med Singapore 2011; 40:237-44

7. Shapira L, Klinger A, Tadir A, Wilensky A, Halabi A Effect of a niobium-containing titanium alloy on osteoblast behavior in culture Clin Oral Implants Res 2009; 20:578-582.

8. Velasco-Ortega E, Jos A, Cameán AM, Pato-Mourelo J, Segura-Egea JJ In vitro evaluation of cytotoxicity and genotoxicity of a commercial titanium alloy for dental implantology Mutat Res 2010; 702:17-23.

9. Bougherara H, Bureau MN, Yahia L Bone remodeling in a new biomimetic polymer-composite hip stem J Biomed Mater Res A 2010; 92:164-174.
10. Sarot JR, Contar CM, Cruz AC, de Souza Magini R Evaluation of the stress distribution in CFR-PEEK dental implants by the three-dimensional finite element method J Mater Sci Mater Med 2010; 21:2079-2085

11. Friedrich RE, Todorovic M, Heiland M, Scheuer HA, Krüll A Scattering effects of irradiation on surroundings calculated for a small dental implant Anticancer Res 2012;32:2043-6.

12. Andreiotelli M, Wenz HJ, Kohal RJ Are ceramic implants a viable alternative to titanium implants? A systematic literature review Clin Oral Implants Res 2009; 20:32-47.

13. Aydin C, Yilmaz H, Ata SO Single-tooth zirconia implant located in anterior maxilla A clinical report N Y State Dent J 2010; 76:30-33.

14. Bilhan H, Bural C, Geckili O Titanium hypersensitivity A hidden threat for dental implant patients? N Y State Dent J 2013; 79:38-43.

15. Hosoki M, Nishigawa K, Miyamoto Y, Ohe G, Matsuka Y Allergic contact dermatitis caused by titanium screws and dental implants J Prosthodont Res 2016;60:213-9

16. Özkurt Z, Kazazoğlu E Zirconia dental implants: a literature review J Oral Implantol 2011; 37:367-376.

17. Özkurt Z, Kazazoğlu E Clinical success of zirconia in dental applications J Prosthodont 2010; 19:64-68.

18. Zhao Y, Wong HM, Wang W, Li P, Xu Z, Chong EY, Yan $\mathrm{CH}$, Yeung $\mathrm{KW}$, Chu PK Cytocompatibility, osseointegration, and bioactivity of three-dimensional porous and nanostructured network on polyetheretherketone Biomaterials 2013 ; 34:9264-77.

19. Najeeb S, Zafar MS, Khurshid Z, Siddiqui F Applications of polyetheretherketone (PEEK) in oral implantology and prosthodontics J Prosthodont Res 2016; 60:12-9.

20. Schwitalla A, Müller WD PEEK dental implants: a review of the literature J Oral Implantol 2013; 39:743-9.

21. Moon SM, Ingalhalikar A, Highsmith JM, Vaccaro AR Biomechanical rigidity of an all-polyetheretherketone anterior thoracolumbar spinal reconstruction construct: an in vitro corpectomy model Spine J 2009; 9:330-335.

22. Rabiei A, Sandukas S Processing and evaluation of bioactive coatings on polymeric implants J Biomed Mater Res Part A 2013; 101:2621-9

23. Barkarmo S, Wennerberg A, Hoffman M, Kjellin P, Breding $\mathrm{K}$, Handa $\mathrm{P}$, et al Nano-hydroxyapatite-coated PEEK 
implants: a pilot study in rabbit bone J Biomed Mater Res Part A 2013; 101:465-71

24. Barkarmo S, Andersson M, Currie F, Kjellin P, Jimbo R, Johansson $\mathrm{CB}$, et al Enhanced bone healing around nanohydroxyapatite-coated polyetheretherketone implants: an experimental study in rabbit bone J Biomater Appl 2014; 29:737-47.

25. Johansson P, Jimbo R, Kjellin P, Currie F, Chrcanovic BR, Wennerberg A Biomechanical evaluation and surface characterization of a nano-modified surface on PEEK implants: a study in the rabbit tibia Int J Nanomed 2014; 9:3903

26. Lai YH, Kuo MC, Huang JC, Chen M On the PEEK composites reinforced by surface-modified nano-silica Mater Sci Eng A 2007; 458:158-69

27. Wu X, Liu X, Wei J, Ma J, Deng F, Wei S Nano-TiO2/ PEEK bioactive composite as a bone substitute material: in vitro and in vivo studies Int J Nanomed 2012; 7:1215-25

28. Suska F, Omar O, Emanuelsson L, Taylor M, Gruner P, Kinbrum A, et al Enhancement of CRF-PEEK osseointegration by plasma-sprayed hydroxyapatite: a rabbit model J Biomater Appl 2014; 29:234-42

29. Poulsson AH, Eglin D, Zeiter S, Camenisch K, Sprecher C, Agarwal Y, et al Osseointegration of machined, injection moulded and oxygen plasma modified PEEK implants in a sheep model Biomaterials 2014; 35:3717-28

30. Wang L, He S, Wu X, Liang S, Mu Z, Wei J, et al Polyetheretherketone/nano-fluorohydroxyapatite composite with antimicrobial activity and osseointegration properties Biomaterials 2014; 35:6758-75.

31. Wheeler SL Eight-year clinical retrospective study of titanium plasma-sprayed and hydroxyapatite-coated cylinder implants Int J Oral Maxillofac Implants 1996; 11:340-50

32. Ha S, Mayer J, Koch B, Wintermantel E Plasma-sprayed hydroxylapatite coating on carbon fibre reinforced thermoplastic composite materials J Mater Sci Mater Med 1994; $5: 481-4$.

33. P G Coelho, G Giro, H S Teixeira et al Argon-based atmospheric pressure plasma enhances early bone response to rough titanium surfaces Journal of Biomedical Materials Research Part A.2012; 100:1901-6.

34. Guastaldi FP, Yoo D, Marin C, Jimbo R, Tovar N, ZanettaBarbosa D, Coelho PG Plasma treatment maintains surface energy of the implant surface and enhances osseointegration Int J Biomater 2013; 2013:354125.
35. H S Teixeira, C Marin, L.Witek et al., Assessment of a chairside argon-based non-thermal plasma treatment on the surface characteristics and integration of dental implants with textured surfaces, Journal of the Mechanical Behavior of Biomedical Materials.2012;9:45-9.

36. P G Coelho, G Cardaropoli, M Suzuki, and J E Lemons, Histomorphometric evaluation of a nano thickness bioceramic deposition on endosseous implants: a study in dogs Clinical Implant Dentistry and Related Research.2009;11: 4292-302

37. D M Dohan Ehrenfest, P G Coelho, B.-S Kang, Y.-T Sul, $\mathrm{T}$ Albrektsson Classification of osseointegrated implant surfaces: materials, chemistry and topography Trends in Biotechnology 2010; 28;198-206

38. T Albrektsson and A.Wennerberg Oral implant surfaces: part 2-review focusing on clinical knowledge of different surfaces International Journal of Prosthodontics.2004;17: 544-564.

39. T Albrektsson, D Buser, and L Sennerby On crestal/ marginal bone loss around dental implants The International Journal of Oral \& Maxillofacial Implants.2012;27: 736-738.

40. Roth JR Industrial plasma engineering Bristol, Philadelphia: The institute of physics publishing; 2000

41. Danna NR, Beutel BG, Tovar N, Witek L, Marin C, Bonfante EA, Granato R, Suzuki M, Coelho PG Assessment of Atmospheric Pressure Plasma Treatment for Implant Osseointegration Biomed Res Int 2015; 2015:761718.

42. Giro G1, Tovar N, Witek L, Marin C, Silva NR, Bonfante EA, Coelho PG Osseointegration assessment of chairside argon-based nonthermal plasma-treated Ca-P coated dental implants J Biomed Mater Res A 2013; 101:98-103.

43. Noeske M, Degenhardt J, Strudthoff S, Lommatzsch U Plasma jet treatment of five polymers at atmospheric pressure: surface modifications and the relevance for adhesion International Journal of Adhesion \& Adhesives 2004; 24: 171-177.

44. Raines AL, Olivares-Navarrete R, Wieland M, et al Regulation of angiogenesis during osseointegration by titanium surface microstructure and energy Biomaterials 2010; 31:4909-17.

45. Inzunza, C Covarrubias, A Von Marttens, Y Leighton, J.C Carvajal, F.Valenzuela, M Díaz-Dosque, N Méndez, C Martínez, A.M Pino, J.P Rodríguez,M Cáceres, P Smith 
Synthesis of nanostructured porous silica coatings on titanium and their cell adhesive and osteogenic differentiation properties, J Biomed Mater Res A 2014;102: 37-48.

46. D.L Wheeler, M.J Montfort, S.W McLoughlin, Differential healing response of bone adjacent to porous implants coated with hydroxyapatite and 45S5bioactive glass, J
Biomed Mater Res 2001;55: 603-612.

47. M Yamada, T Ueno, N Tsukimura, T Ikeda, K Nakagawa, N Hori, T Suzuki, T Ogawa, Bone integration capability of nanopolymorphic crystalline hydroxyapatite coated on titanium implants, Int J Nanomed 2012;7 :859-873. 